\title{
Probleme einer integrierten Analyse der Entwicklungsdynamiken der Berufsgliederung
}

Der einschneidende ökonomische Strukturwandel und die Notwendigkeit eines gesellschaftspolitischen Kurswechsels machen Datenanalysen erforderlich, mit denen die Differenzierungen und Dynamiken der Berufsgliederung zusammenhängend und vorausschauend abgebildet werden können. Die bisherigen Analysemodelle beschränken sich entweder zu sehr auf begrenzte Einzelentwicklungen oder auf pauschale Angaben zu Globaltrends vertikaler Klassen- oder horizontaler Sektorstrukturen. Durch die Herausforderungen neuerer Strukturverschiebungen, insbesondere zur Hochqualifikation, Tertiarisierung, Feminisierung und Prekarisierung, ist jedoch eine Weiterentwicklung der makrosoziologischen Schichtungs- und Klassenmodelle in Richtung einer differenzierten Gesamtanalyse angestoßen worden.

\section{Probleme der Konzept- entwicklung}

Die Entwicklung eines Analysemodells, das die heutigen Differenzierungen und Veränderungen der Berufsgliederung zusammenhängend abbilden kann, steht vor großen Problemen. Sie ist zwar technisch möglich, da heute umfassende Daten und Aufschlüsselungen der Einzelberufe nach internationalen und nationalen Klassifikationssystemen bereitstehen, mit denen das Gefüge der Arbeitsteilung in Deutschland, im internationalen Vergleich sowie im zeitlichen Längsschnitt rationeller untersucht werden könnte. Jedoch haben die Institutionen, die für die Beobachtung des Arbeitsmarktes und der Berufsgliederung zuständig sind, für eine solche integrierte Gesamtanalyse bisher kein geeignetes Analyse- und Rechenmodell. Die verfügbaren Modelle zur Analyse der Massendaten sind entweder zu stark segmentiert oder zu undifferenziert.

Die statistischen Ämter wie auch die Arbeitssoziologie und die Wirtschaftswissenschaften können mit eigenen Berechnungen bisher nur Teilentwicklungen und nicht Gesamtentwicklungen darstellen. Makrosoziologische Modelle, an die eine integrierte Gesamtanalyse anknüpfen kann, sind bisher nur in der Klassen- und Schichtungssoziologie entwickelt worden.
Diese hat eigene Gliederungskonzepte kreiert, weil sie sich zwar auf die amtliche Berufsstatistik stützt, aber deren Klassifikationssysteme für ihre Fragestellungen nicht unmittelbar verwenden kann.

Die Klassifikationssysteme der amtlichen Statistik sind eng an die Aufgabe gebunden, für die Steuerverwaltung bestimmte sozialversicherungsrechtliche Berufskategorien und für spezifische Einzelinteressen bestimmte Berufsfelder und Sektoren zu unterscheiden. Sie sind daher nur begrenzt deckungsgleich mit den Klassifikationsschemata der Schichtungs- und Klassensoziologie, mit denen vor allem ungleiche soziale Lagen, Chancen und Kompetenzen von Individuen und Gruppen in Gesellschaftsordnungen als Ganzem unterschieden werden sollen. Mit dieser Bindung an die Fragen der sozialen Gerechtigkeit ist das Erkenntnisinteresse der Klassen- und Schichtungssoziologie implizit oder explizit auch politisch, d.h. auf die Gesellschafts- und Wirtschaftspolitik bezogen.

Diese Differenz der Perspektiven gilt auch in umgekehrter Richtung. Die herkömmlichen makrosoziologischen Konzepte der sozialen Gliederung können nicht unmittelbar auf die spezifischen Fragen der Arbeitssoziologie, der Arbeitsmarkt- und Wirtschaftsforschung angewandt oder "heruntergebrochen“ werden. Sie können zwar als Ausgangspunkt einer Gesamtanalyse der Arbeitsteilung und Berufsgliederung genutzt werden, bedürfen aber der konzeptionellen Weiterentwicklung. Dazu müssen ihre Erkenntnisinteressen und ihre Dimensionen erweitert und ihre analytischen Unterscheidungen für die arbeits-, berufs- und wirtschaftswissenschaftlichen Fragestellungen stärker differenziert werden.

\section{Weber und Geiger: Strukturelle Differenzie- rung und Dynamik}

Die makrosoziologischen Analysemodelle stützen sich seit ihren Anfängen in der klassischen Soziologie auf eigene, theoretisch fundierte Konzepte der Gliederung der Gesellschaft. In Abgrenzung von ver-

\footnotetext{
Sonja Weber-Menges, Dr., Lehrbeauftragte an der Universität Siegen. Arbeitsschwerpunkte: Sozialstrukturanalyse und soziale Ungleichheit, Bildungssoziologie, Migrationssoziologie, Familiensoziologie. e-mail: weber@soziologie.uni-siegen.de Michael Vester, Dr., Prof. i. R. an der Leibniz Universität Hannover. Leiter der Forschungsgruppe Habitus und Milieu. Arbeitsschwerpunkte: Politische Soziologie und Geschichte sozialer Strukturen, Mentalitäten, Bewegungen und Partizipation. e-mail: m.vester@ipw.uni-hannover.de
} 
einfachenden Klassenmodellen hat schon Max Weber die großen Klassen wegen ihrer inneren Vielfalt in homogenere Einheiten unterteilt. Für die deutsche Klassenstruktur zu Beginn des 20. Jahrhunderts gelangt er zu einer Differenzierung in drei vertikale Klassenstufen, die jeweils wieder in zwei bzw. drei horizontale Fraktionen untergliedert sind (Weber 1980, S. 178f.). Diese Struktur wird durch das zweidimensionale Raumbild, in das wir den Wortlaut der Weberschen Beschreibung übersetzt haben, veranschaulicht (Übersicht 1). Weber macht noch eine zweite, wichtige Unterscheidung: Er entwickelt zwei verschiedene Raumgliederungen, eine für die ökonomisch-berufliche und eine andere für die sozio-kulturelle Handlungsebene der Klassen der Gesellschaft. Die beiden Gliederungen sind nicht gleich, aber doch strukturähnlich oder „homolog“, wie es Pierre Bourdieu (1982, S. 211ff.), der die räumlichen Differenzierungen Webers annähernd vollständig übernommen hat, ausdrückt. Die beiden „Landkarten“ Webers stellen - ähnlich wie etwa die morphologische und politische Landkarte eines Territoriums - zwei Aspekte der gleichen Realität dar. Sie können daher auch, wie dies später Bourdieu (ebd., S. 211) für seine eigenen Raumbilder formuliert, wie auf Transparentpapier gezeichnete Landkarten übereinandergelegt und gelesen werden.

Mit der ersten „Klassenkategorie“, der „Erwerbsklasse“, fasst Weber die Klassen nur von ihrer ökonomischen Seite her, als Produktivkräfte bzw. funktionale Einheiten der gesellschaftlich-beruflichen Arbeitsteilung, ins Auge. In diesen Einheiten, die Weber (1980, S. 177f.) als „Er- werbsklassen“ und Kocka (1980, S. 104) als „ökonomische Klassen“ bezeichnet, sind jeweils ähnliche Einzelberufe zu gröBeren Berufsgruppen zusammengefasst. Die nicht-ökonomische Seite der Klassen bezeichnet Weber mit der zweiten Kategorie, der „sozialen Klasse“, die dem Begriff des „beruflichen Milieus“ bei Emile Durkheim (1988) nahe kommt. Sie umfasst die nach Klassen verschiedene Art der Lebensführung, der Alltagsmentalität und der Strategien der Interessenvertretung, die durch die ökonomische Lage allein nicht vollständig vorherbestimmt sind, sondern sich relativ eigenständig in den Milieus entwickeln. Gemeinsame Klassenlagen stellen nach Weber (1980, S. 177, 531ff.) keine zwingende, sondern „nur eine mögliche (und häufige) Grundlage eines Gemeinschaftshandelns dar", und aus der gleichen Interessenlage können verschiedene Handlungsweisen folgen. Weber wird also nicht zutreffend interpretiert, wenn (wie bei Goldthorpe) das soziale Handeln als Ergebnis einer rationalen Wahl einzig aus der Erwerbsklassenzugehörigkeit abgeleitet wird. Vielmehr müssen die Praxisformen (wie bei Bourdieu) auch unabhängig, als „relativ autonomes“ Feld, untersucht werden.

Webers Raumbild entspricht seiner Entstehungszeit im beginnenden 20. Jahrhundert. In ihm sind die oberen und auch die mittleren Klassen jeweils nach Bildung und Besitz - Bourdieu (1982, S. 212f.) würde sagen: nach kulturellem und ökonomischem Kapital - horizontal in zwei Fraktionen geteilt. Zu erkennen ist weiterhin der überwiegend ständische Charakter der Mittelklassen: Neben den „alten Mittelstand“ des besitzenden Klein- bürgertums war der „neue Mittelstand“ der Angestellten und Beamten getreten, die sich durch ihren besonders gesicherten und geachteten Status deutlich von der „negativ privilegierten “Erwerbsklasse der Arbeiter abheben. Die Arbeiterklasse ist nach Weber (1980, S. 179) primär durch das ökonomische Lohnarbeitsverhältnis, durch geringe Aufstiegschancen und, im Zuge der Automatisierung des Arbeitsprozesses, durch eine abnehmende Differenzierung nach Qualifikationen definiert.

Weber liefert damit primär „Kategorien", die auf eine historische Klassenkonstellation abgestimmt sind, mit denen er aber diese Konstellation noch nicht empirisch-inhaltlich untersucht. Entsprechend streift er in seiner Skizze auch die historische Dynamik der gesellschaftlichen Arbeitsteilung nur kurz, indem er auf die Homogenisierung und Dequalifizierung der Mehrheit der Arbeiterklasse anspielt, die er, wie Marx, als Folge der Automatisierung des Arbeitsprozesses erwartet (ebd.).

Theodor Geiger, der letzte Vertreter der klassischen Soziologie, verwirft diese Annahme. Er gelangt dazu, indem er zwar, wie Bourdieu, an Webers kategoriale Unterscheidungen der Klassengliederung anknüpft, aber diese theoretisch und methodologisch entscheidend - und auch über Bourdieu hinausgehend - weiterentwickelt. Weber und in seiner Nachfolge auch Bourdieu und Goldthorpe haben Konzepte, mit denen sie die in einer bestimmten Zeit vorgefundene Klassengliederung und das Handeln in ihnen beschreiben können. Doch diese Beschreibungen bleiben statisch. Sie haben keine Konzepte, mit denen sie den historischen Wandel solcher beruflichen und gesellschaftlichen Gliederun-

\section{Übersicht 1: „Klassenkategorien" nach Weber* (Raumbild)}

Die vertikale und die horizontale Gliederung der Gesellschaft nach „Erwerbsklassen " und nach "sozialen Klassen“

\begin{tabular}{|c|c|c|c|c|c|}
\hline \multicolumn{4}{|c|}{ „Erwerbsklassen“ [ökonomische Klassen] } & \multicolumn{2}{|c|}{ "Soziale Klassen“ [Klassenmilieus] } \\
\hline $\begin{array}{l}\text { „Positiv privilegierte } \\
\text { Erwerbsklassen sind } \\
\text { typisch ... }\end{array}$ & $\begin{array}{l}\text {... Unternehmer: } \\
\text { Händler, Reeder, } \\
\text { gewerbliche } \\
\text { Unternehmer, } \\
\text { landwirtschaftliche } \\
\text { Unternehmer, Bankiers } \\
\text { und Finanzierungs- } \\
\text { unternehmer, ... }\end{array}$ & $\begin{array}{l}\text {... unter Umständen: } \\
\text { mit bevorzugten } \\
\text { Fähigkeiten oder } \\
\text { bevorzugter Schulung } \\
\text { ausgestattete, freie } \\
\text { Berufe' (Anwälte, } \\
\text { Ärzte, Künstler), ... }\end{array}$ & $\begin{array}{l}\text {... Arbeiter mit } \\
\text { monopolistischen } \\
\text { Qualitäten (eigenen } \\
\text { oder gezüchteten oder } \\
\text { geschulten)." }\end{array}$ & $\begin{array}{l}\text { „die Klassen der } \\
\text { Besitzenden ... }\end{array}$ & ... und durch Bildung Privilegierten" \\
\hline „als ,Mittelklassen“ ... & $\begin{array}{l}\text {... die selbstständigen } \\
\text { Bauern und } \\
\text { Handwerker. }\end{array}$ & $\begin{array}{l}\text { Ferner sehr oft: } \\
\text { Beamte (öffentliche } \\
\text { und private)" ', 'die } \\
\text {,freien Berufe'"“" und }\end{array}$ & $\begin{array}{l}\text {... die "Arbeiter mit } \\
\text { [...] monopolistischen } \\
\text { Qualitäten" }\end{array}$ & $\begin{array}{l}\text { "das Kleinbürger- } \\
\text { tum } \ldots\end{array}$ & $\begin{array}{l}\text {... und die besitzlose Intelligenz und } \\
\text { Fachgeschultheit (Techniker, kommerzielle } \\
\text { und andere ,Angestellte', das Beamtentum, } \\
\text { untereinander evtl. sozial sehr geschieden, je } \\
\text { nach Schulungskosten)“ }\end{array}$ \\
\hline $\begin{array}{l}\text { "Negativ privilegierte } \\
\text { Erwerbsklassen sind } \\
\text { typisch Arbeiter ... }\end{array}$ & $\begin{array}{l}\text {... in ihren } \\
\text { verschiedenen } \\
\text { qualitativ besonderen } \\
\text { Arten: gelernte, ... }\end{array}$ & ... angelernte, & ...ungelernte. ${ }^{\prime \prime}$ & \multicolumn{2}{|c|}{$\begin{array}{l}\text { „die Arbeiterschaft als Ganzes, je automatisierter der Arbeitsprozess } \\
\text { wird“ }\end{array}$} \\
\hline
\end{tabular}


gen als Ganzes theoretisch und empirisch fassen können. Geiger entwickelt solche Konzepte, indem er Webers Kategorien mit Inhalt füllt, in seinen großen Analysen über die soziale Schichtung des deutschen Volkes am Vorabend des Dritten Reiches (Geiger 1932) und über den weiteren Wandel der Klassenstrukturen im wohlfahrtsstaatlich regulierten Kapitalismus (Geiger 1949).

Geiger hat als Erster das Konzept der beruflichen Arbeitsteilung auf der empirischen Grundlage einer nationalen Berufszählung angewandt und dafür auch eine Untersuchungsmethode entwickelt. Er gruppierte die Berufe völlig neu, da die Klassifikationen der amtlichen Statistik für seine Fragestellung nicht tauglich waren. Mit seinem „aszendierenden“, d. h. vom Einzelberuf zu konsistenteren Struktureinheiten „aufsteigenden“ Verfahren (Geiger 1932, S. 17f.) konnte er als Erster ein integriertes Gesamtbild der enormen Verschiebungen der Sozialstruktur bis in die 1920er Jahre zeichnen, indem er die einzelnen Berufe nach bestimmten Kriterien „von Hand“ neu klassifizierte und schrittweise zu einer räumlichen Gruppengliederung zusammenfasste. In dieser Gliederung ergänzte er die dreistufige „Rohgliederung nach dem Produktionsverhältnis" durch eine fünfteilige (und in sich weiter differenzierte) „Tiefengliederung“ nach ähnlichen „Lagen“ und Formen der „Mentalität“ und des „Habitus“. Dadurch gelangt er, ähnlich wie vor ihm Weber und nach ihm Bourdieu, zu einer vertikalen Stufung in drei Lagen, die wiederum horizontal nach ökonomischem oder kulturellem Kapital differenzierbar sind. ${ }^{1}$ (Geiger 1932, S. 24f.)

Geiger hat mit dieser mehrdimensionalen Schichtstruktur, in der die Schichtlinien sich kreuzen, nicht eine beliebige Pluralität, sondern eine spezifische historische Dynamik von Produktionsweisen vor Augen. Mit dieser Sicht entwickelte er eine Alternative zu der vereinfachenden vulgärmarxistischen These der „Proletarisierung“ und „Verelendung“, nach der die kapitalistischen und proletarischen Kerne der Gesellschaft „als Pol und Gegenpol in einem magnetischen Feld" (Geiger 1949, S. 52) fungieren, welche die Mittelschicht in Lohnarbeiter verwandeln und das Proletariat verelenden lassen. In Wirklichkeit sind, so Geiger, sich entwickelnde Gesellschaften oft „plural“ geschichtet, indem sie sich aus älteren ständischen, modernen klassenge- sellschaftlichen und zukunftswichtigen neuen Elementen der Schichtdifferenzierungen zusammensetzen (ebd., S. 37ff.). So „werden Reste der ständischen Schichtung in der Klassengesellschaft bewahrt sein, und diese wird vielleicht schon die Keime einer abermals neuen Schichtungsstruktur in sich entwickelt haben" (ebd., S. 153). Mit der Annahme, ein neuer Strukturzustand löse einen alten nicht plötzlich ab, sondern müsse sich in ihm „vorbereitet haben und aus ihm hervorwachsen“ (ebd., S. 52), knüpft Geiger direkt an Marx' Theorie der historischen Produktionsweisen an. Zusätzlich stellt Geiger (1932, S. 84ff.) fest, dass die hohe Beharrungskraft solcher Formationen zu „sozialgeschichtlichen Verwerfungen“ führen kann, wie sie sich zeigen am Fortwirken von Elementen der mittelalterlich-ständischen Kultur und Wirtschaftsweise im alten bäuerlichen und gewerblichen Mittelstand und bei Teilen des „neuen Mittelstands“ der Angestellten und Beamten, was diese besonders anfällig für die ständisch-autoritäre Nazi-Propaganda machte.

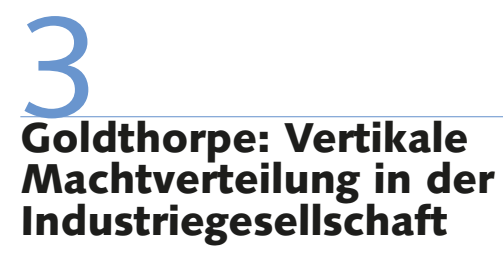

Die heute einflussreichen makrosoziologischen Analysemodelle folgen anderen Fragestellungen, die mit ihrer Entstehung in den 1970er Jahren zusammenhängen. Die Debatten der klassischen Soziologie hatten sich vor allem mit den sozialen Konflikten beschäftigt, die mit dem Übergang zum industriellen Kapitalismus, der Verwandlung der kleinen Selbstständigen in Lohnarbeiter und den Kämpfen um die Durchsetzung und Verteidigung einer parlamentarischen, sozialpolitisch ausgleichenden Demokratie verbunden waren. Diese Konflikte schienen nach 1945 durch den Aufbau einer stabilen politischen Demokratie und einer zunehmenden Teilhabe der arbeitenden Klassen an Wirtschaftswachstum und Wohlfahrtsstaatlichkeit zunehmend bewältigt zu werden. Die Debatten drehten sich daher um die Frage, ob mit dem demokratischen Wohlfahrtsstaat in den modernen Industriegesellschaften die alten vertikalen Klassenteilungen „nivelliert" und die Arbeiter „verbürgerlicht“ seien (vgl. Schelsky 1965; Marcuse 1967).
Hinzu kam in den 1970er Jahren die Debatte, ob und inwieweit die industrielle Klassengesellschaft zunehmend durch eine „postindustrielle Dienstleistungsgesellschaft" abgelöst werde (Touraine 1971; Bell 1985).

In der Auseinandersetzung mit diesen Thesen entwickelte sich seit den 1960er Jahren eine Gegenposition, die sich nicht zuletzt auf Weber und teilweise auf die an Weber anknüpfende These der industriellen Klassengesellschaft von Ralf Dahrendorf (1957) berief. Insbesondere John Goldthorpe (Erikson et al. 1979), Pierre Bourdieu (1982) und Eric Olin Wright (1985) entwickelten Gegenkonzepte der Klassengliederung, mit denen, auf verschiedene Weise, der Fortbestand vertikaler Klassenteilungen empirisch untersucht und auch weitgehend bestätigt werden konnte. Die Berufung aller dieser Ansätze auf Weber ist insoweit berechtigt, als alle von Webers Konzept der Erwerbsklassen ausgehen und die Einzelberufe nach bestimmten Kriterien zu ökonomischen Erwerbsklassen und zu räumlichen Gesamtgliederungen zusammenfassen. Aber nur Bourdieu übernimmt auch die darüber hinausgehenden kategorialen Differenzierungen Webers vollständig. Goldthorpe wie auch Wright entwickeln rein vertikale Erwerbsklassenmodelle und lassen die Differenzierung in horizontale Klassenfraktionen und die lebensweltliche, nicht-ökonomische Ebene der Klassen weg.

Die gewonnenen Gesamtbilder einer industriellen Klassengesellschaft sind bei allen drei Modellen zu wenig differenziert und zu wenig auf die neuen, dynamischen Differenzierungen der gesellschaftlichen Arbeitsteilung abgestimmt. Diese Engführung und auch das vollständige Vorbeigehen an Geigers Strukturwandelanalyse lassen sich aus dem zeitgebundenen Erkenntnisinteresse ihrer Verfasser erklären: Es ging um den Nachweis des Fortbestehens vertikaler Klassenteilungen.

Von den drei Modellen hat sich das von Goldthorpe in der internationalen Großdatenforschung durchgesetzt, nicht zuletzt auch aus dem technischen Grund,

\footnotetext{
Die oberste, , kapitalistische " Lage ist nicht unterteilt; der "mittleren Lage" werden die "mittleren und kleinen Unternehmer" sowie die "Lohn- und Gehaltsbezieher höherer Qualifikation" zugeordnet, der "proletarischen Lage“ die „Tagewerker auf eigene Rechnung" und die "Lohn- und Gehaltsbezieher minderer Qualifikation" (Geiger 1932, S. 24).
} 


\begin{tabular}{|c|c|c|}
\hline \multirow[t]{2}{*}{ Dienst-Verhältnis } & I & $\begin{array}{l}\text { ("Obere Dienstklasse":) Angehörige der akademischen freien Berufe, leitende Angestellte und höhere Beamte (einschl. der } \\
\text { Hochschul- und Gymnasiallehrer); Manager in großen Industrieunternehmen; große und mittlere selbstständige Unternehmer }\end{array}$ \\
\hline & II & $\begin{array}{l}\text { („Untere Dienstklasse“:) Angehörige von Semiprofessionen und des mittleren Managements, Beamte im mittleren und } \\
\text { gehobenen Dienst; Angehörige gehobener technischer Berufe; Manager in kleinen Industrieunternehmen; Vorgesetzte von } \\
\text { Dienstleistungsbeschäftigten }\end{array}$ \\
\hline \multirow[t]{6}{*}{ Zwischenstellung } & Illa & (Zu „Routine-Dienstleistungen“:) Gehobene einfache Dienstleistungsberufe in Handel und Verwaltung \\
\hline & IIIb & (Zu „Routine-Dienstleistungen“:) Ausführende einfache Dienstleistungs- und Verkaufsberufe \\
\hline & IVa & (Zum „Kleinbürgertum“:) Kleine selbstständige Eigentümer und Handwerker mit Beschäftigten \\
\hline & $\mathrm{IVb}$ & (Zum „Kleinbürgertum“:) Kleine selbstständige Eigentümer und Handwerker und kleine Landwirte ohne Beschäftigte \\
\hline & IVc & $\left(\right.$ ("Landwirte $\left.{ }^{\mu}:\right)$ Landwirte und Kleinbauern; andere selbstständige Werktätige in der primären Produktion \\
\hline & V & (Zu „Arbeiter/Arbeiterklasse“:) Ausführende technische Berufe; Vorarbeiter, Meister, Aufsichtspersonen im Bereich manueller Arbeit \\
\hline \multirow{3}{*}{$\begin{array}{l}\text { Lohnarbeits- } \\
\text { verhältnis }\end{array}$} & VI & (Zu „Arbeiter/Arbeiterklasse“:) Facharbeiter \\
\hline & VIIa & (Zu „Arbeiter/Arbeiterklasse“:) An- oder ungelernte Arbeiter (außer in der Landwirtschaft) \\
\hline & VIIb & (Zu „Arbeiter/Arbeiterklasse" ${ }^{\prime}$ ) Landarbeiter und andere Arbeiter in der primären Produktion \\
\hline
\end{tabular}

dass es besonders praktikabel war: Es war durch die rein vertikale Einordnung in Machthierarchien relativ unkompliziert, es war rechentechnisch und durch die Verwendung der amtlichen Berufsstatistik relativ problemlos anwendbar und es konnte einen bestimmten Aspekt, die vertikale Machtstufung, nachvollziehbar empirisch abbilden. Die Klasseneinteilung erfolgt nach beruflicher Stellung und der internationalen Standardklassifikation von Berufen (ISCO). Insgesamt werden nach diesem Modell elf Klassen identifiziert (Übersicht 2). Soweit es um die vertikalen Klassenunterschiede geht, hat das Goldthorpe-Modell bis heute seine Stärken unter Beweis gestellt. Mit ihm können die ungleichen Aufstiegschancen und Abstiegswahrscheinlichkeiten in der sozialen Schichtung relativ einfach und verlässlich statistisch gemessen werden. Besondere öffentliche Prominenz hat das Schema erlangt, als mit ihm in der ersten PISAStudie errechnet werden konnte, dass im Jahre 2000 die 15-Jährigen aus der „oberen Dienstklasse“ im Vergleich zu den Kindern von Facharbeitern die 4,28-fache Chance hatten, ein Gymnasium zu besuchen und dass die soziale Trennlinie „zwischen der Arbeiterschicht insgesamt und den übrigen Sozialschichten" verläuft (Baumert/ Schümer 2002, S. 356ff.).

Die Stärke des Modells ist aber auch seine Schwäche. Seine theoretische und methodische Engführung liegt vor allem darin, dass es alle Dimensionen - die Unterschiede nach Bildung, Macht, Sektorzugehörigkeit usw. - in eine einzige Makrodimension, die vertikale Klassenschichtung, einordnet. Das Kriterium, nach dem eine Gruppe vertikal eingeordnet wird, ist relativ konventionell an die amtliche Sta- tistik angelehnt - die Stellung im Beruf (Unternehmer, Kleingewerbe, freie Berufe und Arbeitnehmer) und die vertikale Einstufung der Arbeitnehmer nach dem kontraktuellen Beschäftigungsverhältnis, d.h. danach, ob sie den Status von Angestellten haben (bei dem langfristige Sicherheit und Aufstiegschancen mit einem besonderen Vertrauensverhältnis und Autonomiespielräumen verbunden sind) oder den eher unsicheren Status von Lohnarbeitern (bei dem geringere Qualifikation und Selbstständigkeit mit einer stärkeren Kontrolle durch Lohnanreize und Betriebshierarchien verbunden ist) oder eine Mittelstellung zwischen beiden.

Dieses Bild einer steilen sozialen Pyramide ist den traditionellen Klassenteilungen, in denen eine industrieproletarische Basis einem ständischen Überbau untergeordnet ist, noch sehr nahe, die Weber beschrieben hat (vgl. Übersicht 1) und die bis in die 1950er und 1970er Jahren dominant gewesen sind. Die Unterscheidung zwischen manueller und nichtmanueller Arbeit bzw. Arbeitern und Angestellten entspricht noch der alten vertikalen Teilung zwischen industrieller Fertigung und den höheren Verwaltungstätigkeiten. Für die herkömmlichen industriegesellschaftlichen Strukturen ist denn auch das Goldthorpe-Schema einigermaßen angemessen differenziert. Für die neuen Differenzierungen der Dienstleistungsberufe, in denen sich die zunehmende Erwerbsarbeit der Frauen konzentriert, stellt es aber nur pauschale Kategorien zur Verfügung. Es berücksichtigt insgesamt noch nicht hinreichend, dass die in stärkerem Umfang weibliche Dienstleistungsarbeit nicht zuletzt in untergeordneten und weniger qualifizierten Bereichen expandiert, während die al- ten Statusunterschiede zwischen Arbeitern und Angestellten an Bedeutung verlieren.

Im Schema von Wright (1985, S. 25) und in der Beschreibung von Bourdieu (1982, S. 585ff.) ist es dagegen die Arbeiterklasse, die (mit mehr als $40 \%$ ) als undifferenzierte Sammelkategorie behandelt wird, und zwar vorwiegend negativ, über ihre Defizite an Berufsqualifikation, Einkommenshöhe und Macht. Unberücksichtigt bleibt die neue Entpolarisierung der Beschäftigungsstruktur durch rascher steigende Ausbildungsniveaus auch der Arbeiter seit den 1960er Jahren. Die Autoren stehen mehr oder minder noch der alten These der zunehmenden vereinheitlichenden Fragmentierung und Dequalifizierung (de-skilling) der Arbeiterklasse (Weber 1980; Braverman 1974) nahe, obwohl diese seit Beginn der 1960er Jahre in der lebhaften internationalen Diskussion über die Entstehung einer hochqualifizierten „neuen Arbeiterklasse“ (Mallet 1972; Blauner 1964; Kern/Schumann 1984; Piore/Sabel 1989) infrage gestellt worden ist. Im Vordergrund steht das Erkenntnisinteresse an der vertikalen Dimension, der Ungleichverteilung von Macht- und Rangressourcen.

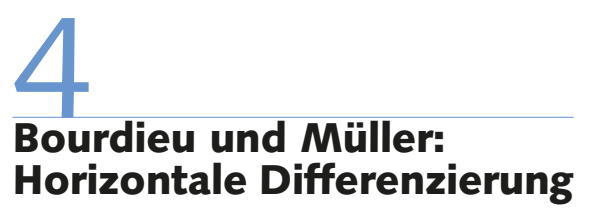

Allerdings hatte Bourdieu begonnen, die vertikale Eindimensionalität der herkömmlichen Klassen- und Schichtkonzepte durch die Einführung einer horizontalen und einer zeitlichen Achse zu durchbrechen (Bourdieu 1982, S. 195ff.). Dieses 
Konzept des dreidimensionalen sozialen Raums wurde schließlich bahnbrechend. Mit seiner Hilfe konnte die Klassenstruktur, die bis dahin als starre Leiter verstanden worden war, als bewegtes räumliches Kräftefeld erschlossen werden. Auf der horizontalen Achse hat Bourdieu vor allem abgebildet, wie sich die Erwerbsklassen durch den zunehmenden Erwerb von Bildungskapital in modernere, besser qualifizierte Fraktionen ausdifferenzierten (ebd., S. 212f.). Der Vorteil des Modells von Bourdieu ist, dass es als mehrdimensionales Achsenmodell die widersprüchlichen Kräfte und Differenzierungen der gesellschaftlichen Arbeitsteilung getrennt abbilden kann. Allerdings ist das Raumkonzept von Bourdieu wenig mehr als eine Skizze, die aus praktischen Gliederungserwägungen entstanden ist. Bourdieu hat weder den Zusammenhang mit der Theorie der gesellschaftlichen Arbeitsteilung noch die empirisch-methodische Operationalisierung mittels eines rechnerischen Konzeptes ausgearbeitet. Dies blieb späteren Entwicklungen überlassen.

Nach Durkheims Theorie hat die gesellschaftliche Arbeitsteilung einen Doppelcharakter (vgl. den Beitrag von Vester in diesem Heft). In der „funktionalen Arbeitsteilung" geht es um die von den Arbeitenden selbst hervorgebrachte berufliche Spezialisierung und Qualifikation. Sie beruht auf Konsensus und Fachkompetenz. In der „erzwungenen Arbeitsteilung“ geht es um die Machtpositionen, die den Arbeitenden durch die Herrschaftshierarchie des Betriebes zugewiesen werden. Sie beruht auf Herrschaft und Statuskompetenz. Unter kapitalistischen Bedingungen, so Durkheim (1988, S. 443ff.), stimmt beides keineswegs überein, was gerade die Ursache des Konfliktes zwischen Unternehmern und abhängig Arbeitenden ist.

Goldthorpes Modell setzt dagegen beide Dimensionen in eins, sodass der möglicherweise nicht beabsichtigte - Eindruck entsteht, dass die unterschiedliche Rangposition der Arbeitenden im Betrieb (und in der Gesellschaft) allein auf Unterschieden der fachlichen Qualifikation beruht. Tatsächlich ist beides ständig in Bewegung; eben dies ist die Ursache des strukturnotwendigen Konfliktes zwischen Unternehmen und Gewerkschaften, der durch die Instrumentarien der Mitbestimmung, der Tarifpolitik, der Betriebs- und Personalräte usw. in der Geschichte der Bundesrepublik immer wieder wesentli- che Struktur- und Machtverschiebungen zustande gebracht hat. Das wesentlich am englischen Pfad mit seinen konservativen Machthierarchien orientierte GoldthorpeModell kann, trotz verschiedener Ansätze der Weiterentwicklung, diese Konfliktstruktur nicht hinreichend abbilden.

Dass Bourdieus Raummodell dies leisten kann, zeigt eine Analyse des Wandels der Erwerbsstruktur der Bundesrepublik zu Beginn der 1990er Jahre (Vester et al. 2001). Die Autoren verbanden Bourdieus Konzept mit Geigers Konzept des Berufsstrukturwandels und Durkheims Konzept der Arbeitsteilung und stellten an den Daten der Berufsstatistik dar, dass sich nicht nur auf den oberen, sondern auch auf den mittleren Hierarchiestufen des Erwerbssystems der Anteil der Beschäftigten der „neuen Berufe“, die sich durch mehr Fachkompetenz und die Notwendigkeit von mehr Mitbestimmung am Arbeitsplatz auszeichnen, von 1950 bis 1987 von rund 5 auf $22 \%$ erhöht hatte (ebd., S. 409). Die Autoren folgerten daraus, dass die mit der horizontalen Dynamik der beruflichen Arbeitsteilung gewachsenen Kompetenzen der Arbeitnehmer zunehmend im Widerspruch zu den von Goldthorpe beschriebenen ökonomischen Machthierarchien stehen, d.h., dass auf den Raumachsen von Bourdieu der klassische Widerspruch zwischen der Dynamik der Produktivkräfte (horizontale Zunahme des kulturellen Kapitals) und der gleichzeitig wirksamen beharrenden Kraft der institutionellen Herrschaftsverhältnisse (vertikale Machtund Chancenhierarchie) abgebildet werden kann. Die abhängig Arbeitenden, die in den rein vertikalen Klassenmodellen bis dahin primär durch ihre Defizite an Ressourcen definiert worden waren, konnten nun auch durch ihre eigenen positiven Ressourcen als immer besser qualifizierte Produktivkräfte und emanzipatorische Gegenmacht beschrieben werden.

Allerdings fehlte noch eine unkompliziert anwendbare Operationalisierung des Raumkonzeptes für die empirische Forschung. Ungelöst war nicht zuletzt das Problem, wie mit der Zunahme der Dienstleistungen, die parallel zur Zunahme des Bildungskapitals zu beobachten war, umzugehen war, die ebenfalls eine Art horizontale Transformation des Erwerbssystems bezeichnete. Für alle neuen Differenzierungen des Erwerbssystems, die sich erst nach den 1970er Jahren voll entfaltet haben - die Kompetenzrevolu- tion, die Tertiarisierung und die Feminisierung - fehlten Analysekonzepte, die sie differenziert genug, im Zusammenhang und als Bewegungen erfassen und abbilden konnten. Die Schwierigkeiten, solche Konzepte zu entwickeln, lagen in der Größe und Heterogenität der Dienstleistungen begründet. Mit heute mehr als zwei Dritteln der Erwerbstätigen und der Wertschöpfung (Geißler 2008, S. 25f.) ist der Dienstleistungssektor eine Rest- und Großkategorie, die Gruppen mit sehr gegensätzlichen Interessen und Handlungslogiken zusammenbindet und daher keine konsistenten Prognosen erlaubt. Insbesondere haben sich die Erwartungen einer Homogenisierung der Dienstleistungen als Sektor, in dem allgemein die belastende, entfremdete und fremdbestimmte abhängige Arbeit verschwinden würde, nicht erfüllt.

Als tragfähig erwies sich schließlich das Konzept, die Kategorie der Dienstleistungen doppelt zu unterteilen, zum einen nach den auf die Berufsausbildung gegründeten vier Qualifikationsstufen und zum anderen, quer dazu, nach den drei grundlegenden qualitativen Segmenten oder Fraktionen der Dienstleistungen. Diese Dreiteilung wurde vor allem von Hanspeter Kriesi (1989) angeregt und wenig später von Walter Müller (1998), dem Partner Goldthorpes in der internationalen Großdatenforschung, auch empirisch erprobt. Müller zerlegte die Residualkategorie der „Dienstleistungen“ in drei horizontale „Segmente“ oder „Klassenfraktionen“, denen er die einzelnen Berufe zuordnete: die „administrative Dienstklasse“, die technischen Experten und die höheren interpersonellen Dienstleistungen. Mit umfassenden Längsschnittanalysen von großen Datensätzen konnte er nachweisen, dass dieser Teilung auch ein unterschiedliches Verhältnis zu Autoritäten und politischen Parteien entsprach. Die Angehörigen der ersten Gruppe, des oberen Managements, waren beruflich an der Einhaltung hierarchischer Autoritätsverhältnisse interessiert und wählten daher überwiegend schwarz-gelb. Die Angehörigen der anderen oberen Klassenfraktionen waren dagegen beruflich an der Autonomie gegenüber Autoritäten interessiert. Müller konnte nachweisen, dass die Zunahme des Rot-Grün-Wählens nicht eine Folge der sog. „Individualisierung“, sondern des Wachstums dieser moderneren Klassenfraktionen war. 


\section{Oesch: Ein mehrdimensio- nales Analysemodell}

Diese und andere Weiterentwicklungen wurden schließlich von Daniel Oesch (2006) in ein neues Analyse- und Rechenmodell integriert und mit einer international vergleichenden Analyse der Berufsgliederung auf der Grundlage von Paneldaten erprobt. Dieser Durchbruch zu einem integrierten neuen Analyse- und Rechenmodell, das die Mängel der herkömmlichen Modelle zu einem großen Teil behebt, wurde dadurch möglich, dass die Logiken, nach denen das Modell zu konstruieren war, neu definiert worden sind.

In seinem Modell kombiniert Oesch das Postulat der Mehrdimensionalität der klassischen Soziologie mit der erprobten technischen Operationalisierung von Goldthorpe. Er verändert das GoldthorpeSchema, indem er die fehlenden Differenzierungsachsen einbezieht. Die vertikale Achse differenziert dabei nach der Höhe des Qualifikationsranges, die horizontale Achse nach den Arbeitslogiken. Für jede Dynamik sieht das Modell damit eine eigene räumliche Differenzierungsachse vor (vgl. Abschnitt 6):

- die Trennlinie zwischen Unternehmern und Arbeitnehmern,

- eine Einteilung nach den vier Stufen des Bildungskapitals (Professionen, SemiProfessionen, Lehrberufe, Un- und Angelernte),

- eine horizontale Dreigliederung der Arbeitnehmerberufe nach drei Arbeitslogiken,

- die Differenzierung der Berufsgruppen nach dem Geschlecht.
Die Unterscheidung nach Arbeitslogiken beruht „zu ungleichen Teilen“ (ebd., S. 69) auf den Arbeiten von Kriesi (1989), EspingAndersen (1993, 1998), Gallie et al. (1998) und Müller (1998). Indem Oesch diese zunächst für die Dienstklasse entworfene Unterscheidung auf die Gesamtheit der Erwerbstätigen anwendet, gelingt es ihm, die heterogene Großgruppe der Dienstleistungen horizontal und vertikal in zehn homogenere Teilgruppen der funktionalen Arbeitsteilung zu unterteilen, über die konsistente Entwicklungs- und Verhaltensprognosen möglich sind. Die Arbeitslogiken werden nach der Organisationsweise des Arbeitsprozesses, nach den Autoritätsbeziehungen, nach der primären Gruppenbindung und nach den Qualifikationsanforderungen unterschieden (Übersicht 3).

Da die Achsen sich überschneiden, erhält Oesch eine strukturierte räumliche Gliederung von 17 Berufsfeldern. Diese sind in sich nach Geschlecht unterteilt und können nach anderen Kriterien wie Region, Branchen, Ethnie usw. zusätzlich unterteilt werden. ${ }^{2}$ Die Raumstruktur ermöglicht es, insbesondere die neuen Dynamiken - Kompetenzsteigerung, Tertiarisierung und Feminisierung - getrennt und in ihrer Interdependenz zu erfassen.

Durch die Integration verschiedener „Arbeitslogiken“ bietet Oeschs Modell, obwohl es von den ökonomischen „Erwerbsklassen“ ausgeht, Anschlussmöglichkeiten zu der zweiten in der klassischen Soziologie unterschiedenen Handlungsebene, zu den lebensweltlichen „sozialen Klassen“. So bildet die Unterscheidung der „Arbeitslogiken“ eine Brücke zum Konzept des „Habitus“ und der „Mentalität“ (Geiger; Bourdieu), zum „Berufsethos“ (Sombart) und zu den „Milieus“ (Durkheim).

Nicht von Bourdieu selbst, aber von vielen Nachfolgestudien konnte die hori- zontale Achse in Bourdieus Konzept des sozialen Raums (Bourdieu 1982, S. 212f.) als die Dimension genutzt werden, auf der sich die historische Entwicklung der Produktivkräfte zur arbeitsteiligen Spezialisierung und Kompetenzakkumulation als Bewegung zum linken Pol des sozialen Raums abbilden lässt (Vester et al. 2007, S. 34f.). Diese räumliche Bewegung ist auch in unserer Darstellung des Oeschschen Modells ${ }^{3}$ erkennbar (vgl. Vester in diesem Heft, Übersicht 1).

\section{Empirisch-methodische Operationalisierung}

Empirisch-methodisch operationalisiert Oesch sein Mosaikbild der 17 Berufsfelder, indem er für vier Vergleichsländer nach

2 Die vier Unterteilungsdimensionen des Modells können aus technischen Gründen in den Übersichten nicht räumlich aufgefaltet, sondern nur zweidimensional wiedergegeben werden.

3 In unserer Darstellung des Oeschschen Raums der Berufsfelder sind die Spalten (die die verschiedenen Arbeitslogiken angeben) nicht in der Reihenfolge von Oesch (2006, S. 68), sondern in der Reihenfolge von Bourdieu (1982, S. 212f.) horizontal nebeneinander angeordnet. Diese Änderung der äußeren Darstellungsform soll die Vergleichbarkeit mit dem Raum der Berufspositionen bei Bourdieu herstellen. Die selbstständigen Unternehmer, bei Oesch ganz links, sind nun am rechten Pol des sozialen Raums von Bourdieu, dem Pol des ökonomischen Kapitals, verortet. Links daneben sind, wegen ihrer Nähe zum Kapital, nun die Management- und Verwaltungsberufe ("organisatorische Logik ") verortet, die bei Oesch ganz rechts eingeordnet sind. Es folgen, von rechts nach links gesehen, daneben die Gruppen der "technischen Arbeitslogik" und der "interpersonellen Arbeitslogik", die bei Oesch ebenfalls anders eingeordnet sind.

\section{Übersicht 3: Dimensionen der Arbeitslogiken der Arbeitnehmerberufe (Raumbild)}

\begin{tabular}{|c|c|c|c|}
\hline & Interpersonelle Dienstleistungslogik & Technische Arbeitslogik & Organisatorische Arbeitslogik \\
\hline $\begin{array}{l}\text { Organisationsform des } \\
\text { Arbeitsprozesses (setting of } \\
\text { work process) }\end{array}$ & $\begin{array}{l}\text { Dienstleistungsbeziehung auf der } \\
\text { Grundlage direkten persönlichen } \\
\text { Austauschs }\end{array}$ & $\begin{array}{l}\text { Durch technische Produktionskriterien } \\
\text { bestimmter Arbeitsprozess }\end{array}$ & Bürokratische Arbeitsteilung \\
\hline $\begin{array}{l}\text { Herrschaftsbeziehungen } \\
\text { (relations of authority) }\end{array}$ & $\begin{array}{l}\text { Arbeit weitgehend außerhalb von } \\
\text { Befehlsstrukturen }\end{array}$ & $\begin{array}{l}\text { Arbeit auf höheren Stufen } \\
\text { eher außerhalb festgelegter } \\
\text { Befehlsstrukturen, auf den unteren } \\
\text { Stufen eher innerhalb dieser }\end{array}$ & $\begin{array}{l}\text { Arbeit innerhalb einer bürokratischen } \\
\text { Befehlshierarchie, die gleichzeitig die } \\
\text { Aufstiegshierarchie ist }\end{array}$ \\
\hline $\begin{array}{l}\text { Primäre Orientierung } \\
\text { (primary orientation) }\end{array}$ & $\begin{array}{l}\text { Orientierung zum Klienten, Schüler, } \\
\text { Patienten oder Bittsteller }\end{array}$ & Orientierung zur Berufsgruppe & $\begin{array}{l}\text { Orientierung zur beschäftigenden } \\
\text { Organisation }\end{array}$ \\
\hline $\begin{array}{l}\text { Qualifikationsanforderungen } \\
\text { (skill requirements) }\end{array}$ & $\begin{array}{l}\text { Fachliches Expertentum und soziale } \\
\text { Kompetenzen auf den höheren Stufen, } \\
\text { soziale Kompetenzen auf den unteren } \\
\text { Stufen }\end{array}$ & $\begin{array}{l}\text { Wissenschaftliches Expertentum auf } \\
\text { höheren Stufen, handwerkliches Können } \\
\text { und manuelle Fertigkeiten auf unteren } \\
\text { Stufen }\end{array}$ & $\begin{array}{l}\text { Koordinations- und Kontrollfähigkeiten } \\
\text { auf den höheren Stufen, qualifizierte } \\
\text { Büroarbeit auf den niedrigen Stufen }\end{array}$ \\
\hline
\end{tabular}




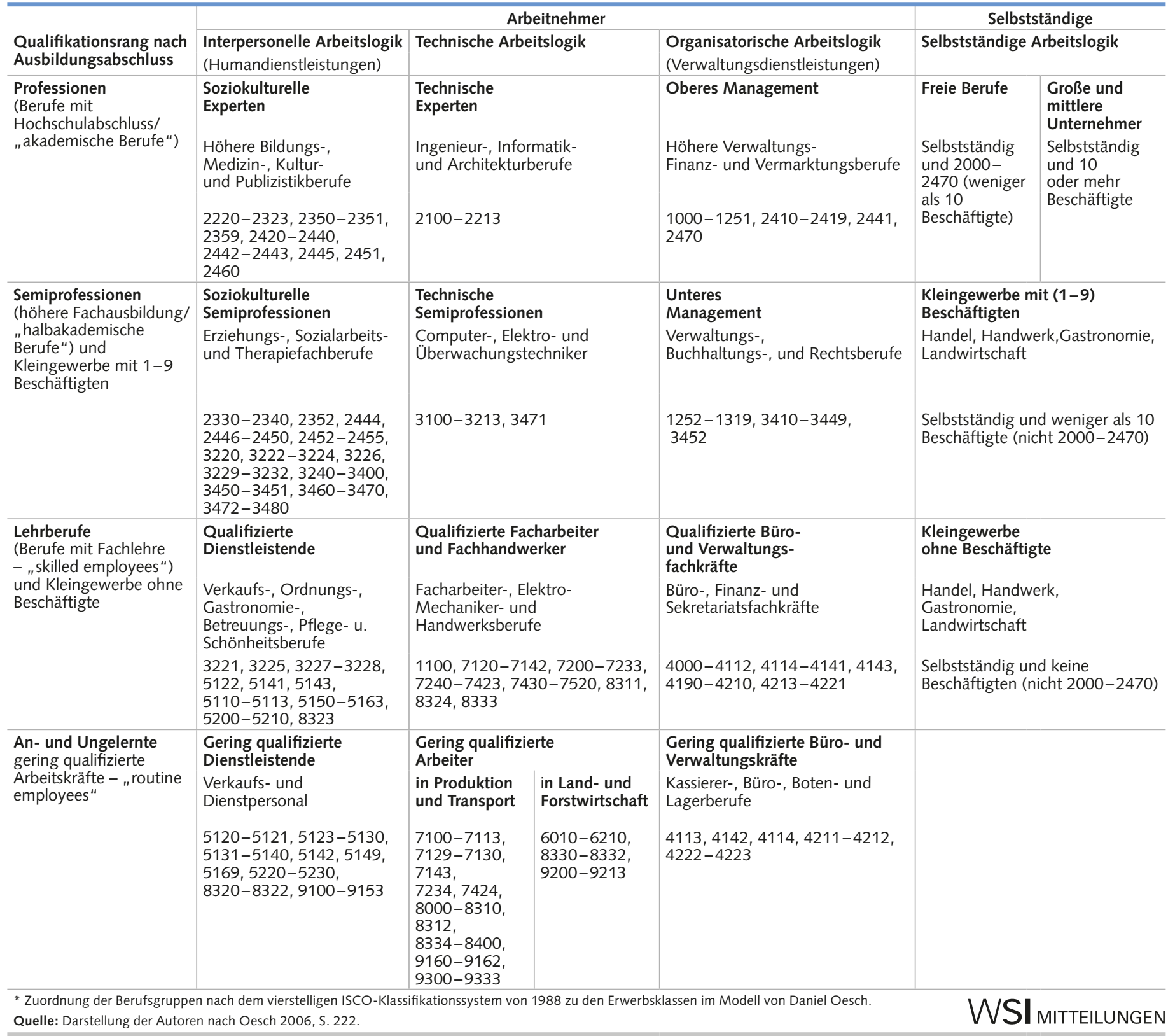

den vierstelligen internationalen Berufskennziffern (ISCO-Kennziffern) ${ }^{4}$ jeden Einzelberuf der Erwerbstätigen einem von 17 Berufsfeldern in seinem mehrdimensionalen Raumbild der Berufsgliederung zuordnet (Übersicht 4).

Um Individuen in das Klassenschema einzuordnen und das Modell von Oesch mithilfe der ISCO-Kennziffern zu operationalisieren (Oesch 2006, S. 79), wurde zunächst die Zielpopulation eingegrenzt und definiert. Zur Zielpopulation gehören ausschließlich Personen zwischen 20 und 65 Jahren, die mindestens 20 Stunden je Woche einer Erwerbstätigkeit nachgehen. Des Weiteren werden Informationen über drei zusätzliche Variablen benötigt:
- Stellung im Beruf, unterschieden nach selbstständigen Unternehmern, freien Berufen, Kleingewerbetreibenden und Arbeitnehmern;

- bei selbstständigen Unternehmern die Zahl der Beschäftigten, wobei die großen Unternehmer (zehn und mehr Beschäftigte), die freien Berufe (ohne Bezug auf die Beschäftigtenzahl), die Kleingewerbetreibenden mit bis zu neun Beschäftigten und die Kleingewerbetreibenden ohne Beschäftigte unterschieden werden;

- Berufsbezeichnung (occupational title), teils ohne und teils mit Berücksichtigung der erworbenen Berufsabschlüsse).
4 Internationale Standard-Klassifikation der Berufe, Fassung 1988, ISCO-88. Anhand es ISCOKlassifikationssystems werden zunächst zehn Hauptberufsgattungen unterschieden: 1. Angehörige gesetzgebender Körperschaften, leitende Verwaltungsbedienstete und Führungskräfte in der Privatwirtschaft; 2. Wissenschaftler; 3 . Techniker und gleichrangige nichttechnische Berufe; 4 . Bürokräfte, kaufmännische Angestellte; 5. Dienstleistungsberufe, Verkäufer in Geschäften und auf Märkten; 6. Fachkräfte in der Landwirtschaft und Fischerei; 7. Handwerks- und verwandte Berufe; 8. Anlagen- und Maschinenbediener sowie Montierer; 9. Hilfsarbeitskräfte; 10. Soldaten. Diese Hauptberufsgattungen werden in Berufsgruppen, Berufsuntergruppen und Berufsgattungen gegliedert, sodass sich jeder Beruf mit einer vierstelligen Codenummer einer Berufsgattung zuordnen lässt. 
Eine schwierige Frage war bei dem dritten Punkt, wie die Dimension der beruflichen Qualifikation behandelt werden kann, um Berufe zu klassifizieren. Für die Klassifizierung sind zunächst die Qualifikationsanforderungen, die mit dem jeweiligen Beruf verbunden sind, von Bedeutung, nicht jedoch die formale Ausbildung des in diesem Beruf Beschäftigten an sich. Qualifikationen sind also nur von Bedeutung, wenn sie im entsprechenden Beruf auch genutzt werden. So muss ein Arzt, der als Monteur tätig ist, zu den gering qualifizierten Arbeitern und nicht zu den soziokulturellen Experten gerechnet werden. Vor allem in westeuropäischen Ländern ist der $\mathrm{Zu}$ sammenhang zwischen Berufsausbildung und ausgeübtem Beruf allerdings besonders stark. Für die Mehrzahl der Berufe erfolgte die Zuordnung der ISCO-Codes in das Schema Oeschs ausschließlich auf der Basis der Berufsbezeichnung. Eine Ausnahme bilden jedoch die Qualifikationsgruppen der Lehrberufe und der Unund Angelernten. Um eine exakte Grenze zwischen qualifizierten Facharbeitern und Fachhandwerkern, Büro- und Verwaltungsfachkräften und Dienstleistenden auf der einen und gering qualifizierten Arbeitern, Dienstleistenden und Büro- und Verwaltungskräften auf der anderen Seite zu ziehen, wurde für diese Beschäftigungsgruppen zusätzlich zur Berufsbezeichnung auch die weitere Variable der Ausbildung berücksichtigt. Diese Vorgehensweise ist auch dazu geeignet, gelernte Fachkräfte in einem bestimmten Beruf von Beschäftigten im selben Beruf zu unterscheiden, die sich die Befähigung zur Ausübung dieser Tätigkeit selbst angeeignet und nicht durch eine Berufsausbildung erworben haben. Ein eher praktischer Vorteil der zusätzlichen Einbeziehung der Ausbildung bei Lehrberufen und Un- und Angelerntenberufen ist der, dass gerade in diesen Bereichen auch im Rahmen der detaillierten vierstelligen ISCO-Codes Berufsbezeichnungen oft zu generell sind, um sie ohne Weiteres im Klassenschema zu verorten.

Ein weiteres Problem besteht darin, dass es in verschiedenen Ländern oft verschiedene Klassifikationen für denselben Beruf gibt. So wird beispielsweise ein Grundschullehrer in Deutschland und England den Professionen zugeordnet, in der Schweiz hingegen den Semiprofessionen. Auch dieser Problematik trägt Oesch Rechnung, indem er zur internationalen Vergleichbarkeit die Zuordnung zu Klassen auf der Basis der Berufsbezeichnung,

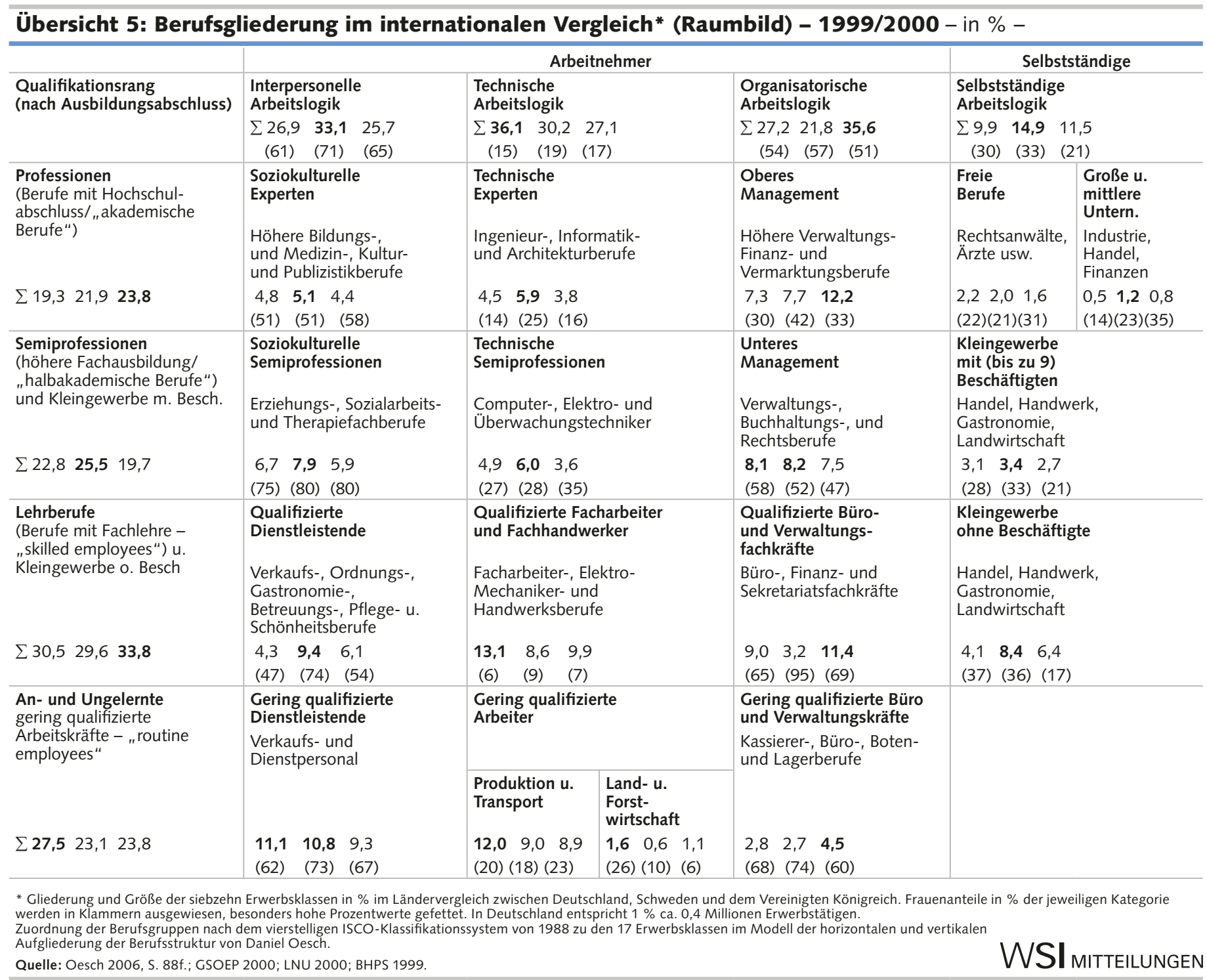


des Socio-Economic Index (SEI) und des Ausbildungsniveaus betrachtet. Oesch bemerkt in diesem Zusammenhang, dass eine Verortung im Schema notwendigerweise auch immer subjektive Beurteilungen beinhaltet.

Die Leistungsfähigkeit des Modells von Oesch liegt primär in der Analyse der Bevölkerungsteile, die erwerbstätig sind. Darüber hinausgehende, in der Erwerbsstatistik unberücksichtigte soziale Teilungen würden eine Erweiterung des Modells erfordern. Nicht berücksichtigt und statistisch erfasst sind insbesondere die Erwerbslosen, die nicht Erwerbstätigen, die sog. Scheinselbstständigen, die nicht erwerbsmäßige Familienarbeit und die Gruppe der Anlage-Kapitalisten, die über das Finanzmarktkapital gut organisiert sind und die nach ihnen benannte neoliberale Politik des "Shareholder-Value“ durchgesetzt haben.

Oesch hat mithilfe seines neuen Konzepts in erster Linie gesellschaftspolitische Fragestellungen untersucht. In seiner 2006 veröffentlichten Untersuchung verwendete er sein Modell, um anhand von Paneldaten um das Jahr 2000 aufzuzeigen, dass die Größenproportionen der Berufsgliederungen von Ländern mit ihren verschiedenen gesellschaftspolitischen Pfaden variieren (vgl. Übersicht 5) und dass auch die parteipolitischen Präferenzen nicht nur mit der vertikalen, sondern auch der horizontalen Erwerbsklassenzugehörigkeit variieren.

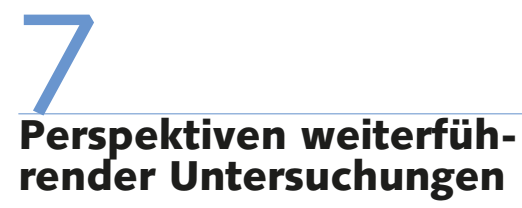

Das Modell von Oesch hat sich darüber hinaus als geeignet erwiesen, weiterführende Analysen der Entwicklung und Differenzierung im Zeitverlauf durchzuführen, die durch den anhaltenden Strukturwandel und die Notwendigkeit eines gesellschaftspolitischen Kurswechsels erforderlich geworden sind. Die in dem Beitrag von Vester in diesem Heft vorgestellte begrenzte Pilotuntersuchung hat diese Eignung nachgewiesen und ist in ihrer bisherigen Reichweite auch geeignet, grundlegende Hinweise zu den berufsstrukturellen Implikationen eines gesellschaftspolitischen Pfadwechsels zu geben.

Gleichzeitig hat die Pilotuntersuchung aber auch deutlich gemacht, dass durch differenziertere und erweiterte Längsschnittanalysen mit größeren Datensätzen noch weit mehr Zusammenhänge der Dynamiken der nationalen und nicht zuletzt der internationalen beruflichen Arbeitsteilung aufgedeckt werden müssten und könnten. Für solche vertiefenden Analysen kann und müsste das Analysemodell Oeschs weiterentwickelt werden. Es geht hierbei vor allem um das Hinzufügen neuer Differenzierungen nach Branchen, bestimmten Berufsgruppen, Regionen usw. sowie auch um feinere Aufschlüsselungen nach Geschlecht, Alter, Migrationshintergrund, Bildungsabschlüssen, Qualifikationserfordernissen am Arbeitsplatz, Einkommen, Arbeitszeit und Arbeitsvertragsform sowie nach weiteren Fragestellungen der Arbeitssoziologie und Wirtschaftswissenschaften. Für diese Differenzierungen ist die Stichprobe des Sozio-oekonomischen Panels (SOEP), mit der Oesch und eine Pilotstudie für die IG Metall (Vester et al. 2009) auf der Grundlage der vierstelligen ISCO-Berufskennziffern gearbeitet haben, mit ca. 11.000 Fällen von Erwerbstätigen zweifellos noch zu klein. Geeignet und verwendbar ist jedoch die große Stichprobe des Mikrozensus. Dafür ist das von Oesch entwickelte Analyseprogramm geeignet, zumal es durch spezifische Unterdifferenzierungen verfeinert werden kann.

\section{LITERATUR}

Bell, D. (1985) [1975]: Die nachindustrielle Gesellschaft, Frankfurt a. M./New York

Blauner, R. (1964): Alienation and Freedom - The Factory Worker and His Industry, Chicago/London

Bourdieu, P. (1982) [1979]: Die feinen Unterschiede, Frankfurt a. M. Braverman, H. (1974): Labor and Monopoly Capitalism. The Degradation of Work in the Twentieth Century, New York

Baumert, J./Schümer, G. (2002): Familiäre Lebensverhältnisse, Bildungsbeteiligung und Kompetenzerwerb im internationalen Vergleich, in: Baumert, J. et al. (Hrsg.): PISA 2000: Die Länder der Bundesrepublik Deutschland im Vergleich, Opladen, S. 159-202

Dahrendorf, R. (1957): Soziale Klassen und Klassenkonflikt in der industriellen Gesellschaft, Stuttgart

Durkheim, E. (1988) [1893/1902]: Über soziale Arbeitsteilung, Frankfurt a. $M$.

Erikson, R./Goldthorpe, J. H./Portocarero, L. (1979): Intergenerational Class Mobility in Three Western European Societies: England, France and Sweden, in: British Journal of Sociology 30 (4), S. 341-415

Erikson, R./Goldthorpe, J. H. (1993): The Constant Flux, Oxford Esping-Andersen, G. (Hrsg.) (1993): Changing Classes. Stratification and Mobility in Post-Industrial Societies, London
Esping-Andersen, G. (1998): Die drei Welten des Wohlfahrtskapitalismus. Zur Politischen Ökonomie des Wohlfahrtsstaates, in: Lessenich, St./Ostner, I. (Hrsg.) (1998): Welten des Wohlfahrtskapitalismus, Frankfurt a. M., S. 16-56

Gallie, D./White, M./Cheng, Y./Tomlinson, M. (1998): Restructuring the Employment Relationship, Oxford

Geiger, Th. (1932): Die soziale Schichtung des deutschen Volkes, Stuttgart

Geiger, Th. (1949): Die Klassengesellschaft im Schmelztiegel, Köln/ Hagen

Geißler, R. (2008): Die Sozialstruktur Deutschlands, Wiesbaden

Kern, H./Schumann, M. (1984): Das Ende der Arbeitsteilung? Rationalisierung in der industriellen Produktion: Bestandsaufnahme, Trendbestimmung, München

Kocka, J. (1980): The study of social mobility and the formation of the working class in the $19^{\text {th }}$ century, in: Le Mouvement Social 111 (2), S. $97-118$

Kriesi, H. (1989): New Social Movements and the New Class in the Netherlands, in: American Journal of Sociology 94 (5), S. 1078-1116 Mallet, S. (1972) [1963]: Die neue Arbeiterklasse, Neuwied/Berlin 
Marcuse, H. (1967) [1964]: Der eindimensionale Mensch. Studien zur Ideologie der fortgeschrittenen Industriegesellschaft, Darmstadt/ Neuwied

Müller, W. (1998): Klassenstruktur und Parteiensystem. Zum Wandel der Klassenspaltung im Wahlverhalten, in: Kölner Zeitschrift für Soziologie und Sozialpsychologie (KZfSS) 50 (1), S. 3-46

Oesch, D. (2006): Redrawing the Class Map. Stratification and Institutions in Britain, Germany, Sweden and Switzerland, Basingstoke Piore, M. J./Sabel, Ch. F. (1989) [1984]: Das Ende der Massenproduktion: Studie zur Requalifizierung der Arbeit und die Rückkehr der Ökonomie in die Gesellschaft, Berlin

Schelsky, H. (1965) [1954]: Die Bedeutung des Schichtungsbegriffes in der Analyse der gegenwärtigen deutschen Gesellschaft, in: ders.: Auf der Suche nach der Wirklichkeit, Düsseldorf/Köln
Touraine, A. (1971) [1969]: The Post-industrial Society, New York Vester, M./von Oertzen, P./Geiling, H./Hermann, Th./Müller, D. (2001): Soziale Milieus im gesellschaftlichen Strukturwandel. Zwischen Integration und Ausgrenzung, Frankfurt a. $M$.

Vester, M./Teiwes-Kügler, Chr./Lange-Vester, A. (2007): Die neuen Arbeitnehmer. Zunehmende Kompetenzen - wachsende Unsicherheit, Hamburg

Vester, M./Gardemin, D./Groh-Samberg, O. (2009): Zwischenergebnisse der Berufsfeldanalyse nach Oesch auf der Grundlage des Sozioökonomischen Panels (SOEP) 1990-2007 (Forschungsbericht), Hannover

Weber, M. (1980) [1921]: Wirtschaft und Gesellschaft, Tübingen Wright, E. O. (1985): Classes, London 